\title{
Network Solutions for the LOS Problem of New Indoor Free Space Optical System*
}

\author{
Zeyu Wu and T.D.C. Little \\ Department of Electrical and Computer Engineering \\ Boston University, Boston, Massachusetts \\ $\{$ bobtail,tdcl\}@bu.edu
}

May 11,2010

MCL Technical Report No. 05-11-2010

\begin{abstract}
Recent developments in solid-state light-emitting diode (LED) materials and devices is driving a resurgence into the use of free-space optics (FSO) for wireless broadband communication. This technology uses the visible spectrum provided by "white" LEDs that are becoming ubiquitous in lighting and has some desirable properties competitive with existing radio frequency (RF) communications. By leveraging the low-cost nature of LEDs and lighting units there are many opportunities to exploit this medium for widespread optical communication deployment. The optical medium, however, has particular characteristics, including directionality and susceptibility to noise sources in the visible spectrum that must be managed.

In this paper we present a new indoor FSO system, also known as a visible light communication (VLC) system that addresses achieving satisfactory data rates while supporting multiple access under line of sight (LOS) constraints. A hexagonal physical device design is proposed and investigated in the context to two communication protocols designed to manage point-to-point and point-to-host cases. Theoretical analysis and simulation of the two protocols under the hexagonal transceiver device design indicate suitability for addressing high data rate communications between peer devices; or, via relay, between multiple devices using the peer-to-host model.
\end{abstract}

* In Proc. IEEE, IET International Symposium on Communications Systems, Networks, and Digital Signal Processing, 2010, Newcastle, UK, July 2010. This material is based upon work supported by the National Science Foundation under Grant No. EEC-0812056. Any opinions, findings, and conclusions or recommendations expressed in this material are those of the author(s) and do not necessarily reflect the views of the National Science Foundation. 


\section{Introduction}

RF communication is an incumbent and evolving technology that will have high utility for the indefinite future. However, there are both opportunities with the use of free space optical spectrum and some limitations on the use of RF. RF suffers from several constraints that prevent it from being used in certain scenarios. For next generation of wireless communication technologies, with the development of new laser diodes (LD) and LED materials, researchers [6] believe that FSO presents a viable and promising supplemental technology to the RF system by enabling the use for short range indoor applications in addition to previous outdoor long range cases. Infrared (IR) applications continue to predominate for niche applications (e.g., TV remote controls). Nowadays, due to the development of new energy-efficient LED materials and devices, replacing old incandescent and fluorescent lights with "white" LED lights will undoubtedly happen in the future [1]. These small and power-efficient devices give rise to more interesting wireless communication applications for both indoor and outdoor scenarios as a medium for modulated FSO communications. Researchers are attracted by the opportunities here because of the low-cost and volume production of LED devices for lighting [2-6].

There are many existing demonstrations of FSO communication systems using visible light. Pang et al. constructed a system with visible LEDs for traffic light-based communications in 1999 [7]. The group set up the system with 441 red ultra-bright LEDs in the lab over 20 meters. The system can achieve a rate at $128 \mathrm{kbps}$.

The prototype developed by Douseki et al. [8] is a indoor application for communication within a range of $40 \mathrm{~cm}$ deployed as a desktop lamp without batteries. Power is derived from a solar cell which also acts as a photon detector for receiving data. This prototype can support transmission up to $100 \mathrm{kbps}$ under illumination at the distance $40 \mathrm{~cm}$.

The prototype described by Wada et al. [9] is an extension of a pixelated system [10] in a longrange outdoor application. It uses a LED array for traffic light as a transmitter and a high speed camera as a receiver. The authors claim it can achieve a speed of $2.78 \mathrm{kbps}$ within $4 \mathrm{~m}$ under laboratory conditions.

At the University of Oxford, Minh et al. have developed a prototype [11] that can achieve 100 Mbps. However, currently it only works for a very short distance $(10 \mathrm{~cm})$.

Little et al. at Boston University demonstrated a short range $(3 \mathrm{~m})$ duplex point-to-point whiteLED system with the rate of $56 \mathrm{kbps}$ [12] developed with readily-available electronics and LEDs, demonstrating the viability, simplicity, and low cost of VLC solutions rather than their upper bound in terms of achievable data rates. The same team created a prototype that delivers in excess of 1 Mbps while providing both illumination and communication at several meters and has been demonstrated as an array of seven luminaries in the form of overhead spot lighting.

However, like every other new technology, FSO communication using visible light is in the early stage of development and has many problems or limitations that need to be solved. One of them is signal occlusion of the LOS channels.

Although visible light is more able to be reflected due to its larger refractive index than IR, both still suffer from path loss that can make the receive signal-to-noise ratio (SNR) very poor. Multi-Spot Diffusing (MSD) [13] provides a solution by beaming the signal to the ceiling to form several reflected light sources with Lambertian illumination pattern. However, the source needs to be located at a desktop level, and fixed to provide stable light sources. In our work, and in this paper, we introduce two network solutions for this problem through the use of relays for data 
through other nodes or hosts.

The paper is organized as follows. In Section 2, the model of our system is introduced with performance parameters. The details of our network solutions are provided in Section 3. Simulation and theoretical analysis are discussed in Section 4. Section 5 concludes the paper.

\section{The FSO System Model}

The model for our proposed FSO system with support of multiple access and mobility is illustrated in Fig. 1.

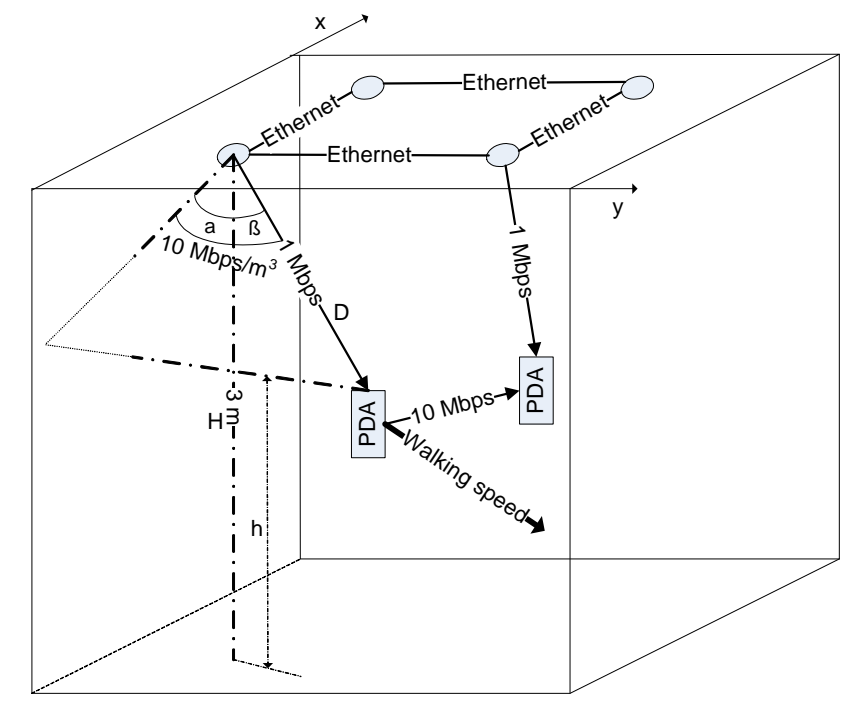

Figure 1: Proposed FSO system model for indoor applications

In this system, we assume a basic transmit rate of the transmitter of $10 \mathrm{Mbps}$ with a distance up to approximately $3 \mathrm{~m}$. From the host, the total speed can be satisfied in a unit cubic meter area is $10 \mathrm{Mbps} / \mathrm{m}^{3}$. When multiple access is supported, the speed of downlink per user can be up to 1 Mbps under the satisfaction of the total rate requirement. The device on the user side should be able to support mobility without sacrificing this performance.

We adopt a device in the form of a hexagonal cylinder shown in Fig. 2 for the desktop level user device. The original idea is a honeycombed sphere as proposed by [14]. However, this design is not suitable for us. First, despite the circuit, if we put 10s of LEDs on each face, hundreds of LEDs are required such that the size and cost will be impractically large for conventional LEDs. Second, in our system, the faces are assigned to two jobs explicitly. For honeycombed sphere, there are faces with field of view (FOV) between horizontal and vertical. Therefore, whichever job we assign to them, they will cause interference to the faces assigned with the other job.

In our ultimate system, additional features will be included that are not investigated here in this paper, including ad hoc solutions for LOS requirement, multiple access control and exploring the feasibility of using OFDM with other signaling techniques.

The top face, which is responsible for the communication with the base station, is quite different from the rest of the faces. If the white light from lamp consists of red, green and blue, we can equip the receivers with one, two or three different optical filters for different colors as indicated in 


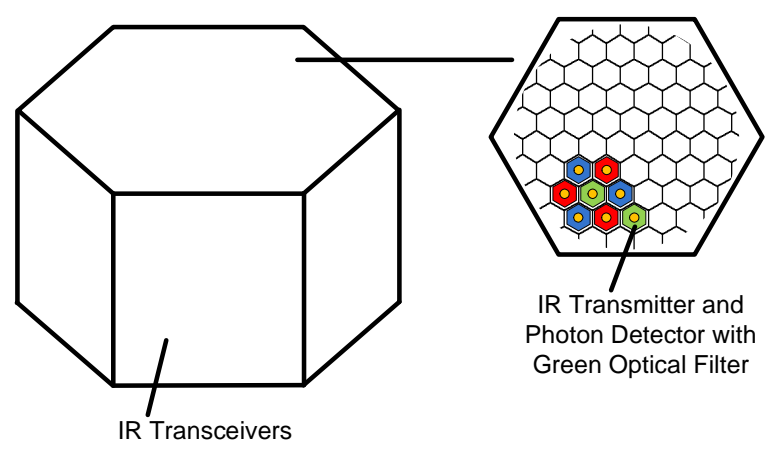

Figure 2: Desktop level user device

Fig. 2. This is for the purpose of exclusively receiving of different services or achieving multiple communication channels for high data rate.

The rest of the faces are responsible for communication with other user devices. Since the transceivers on each face are independent from those on other faces, simultaneous communication can be enabled between multiple user devices. Furthermore, since the 360 degree omni-direction is covered by multiple faces, the link model is approximately point-to-point (quasi-point-to-point) and the FOV is much narrower than the top face. This advantage can greatly reduce multipath distortion and background light noise so that the transceiver design is much simpler. However, angle diversity is achieved with the expense of spatial reuse.

Another advantage of the user device is that it can support mobility and solve the non-LOS blocking problem which is especially important for point-to-point link model. The details of which will be covered later.

\section{Proposed Solutions for LOS Blocking}

Because of the inherent property of light mentioned before, LOS is required to provide continuous connectivity. Although signal reflection still exists, this technology suffers from a high path loss due to the absence of a direct path and data-rate is greatly limited. Based on the system model we introduced, there are two possible solutions for this problem.

\subsection{Peer-to-Peer Protocol}

The first protocol achieves the goal of solving blocking by exploring the possibility of node-tonode communication among user devices. Basically, when blocking happens between two nodes, the source node will begin a search procedure through other nodes in the network to find a multihop path. The procedure is introduced as follows:

\subsection{1}

When connection between two nodes is interrupted, the source node will first check all other faces that if destination node exists in the LOS of any of them. If yes, nodes can reestablish the link 


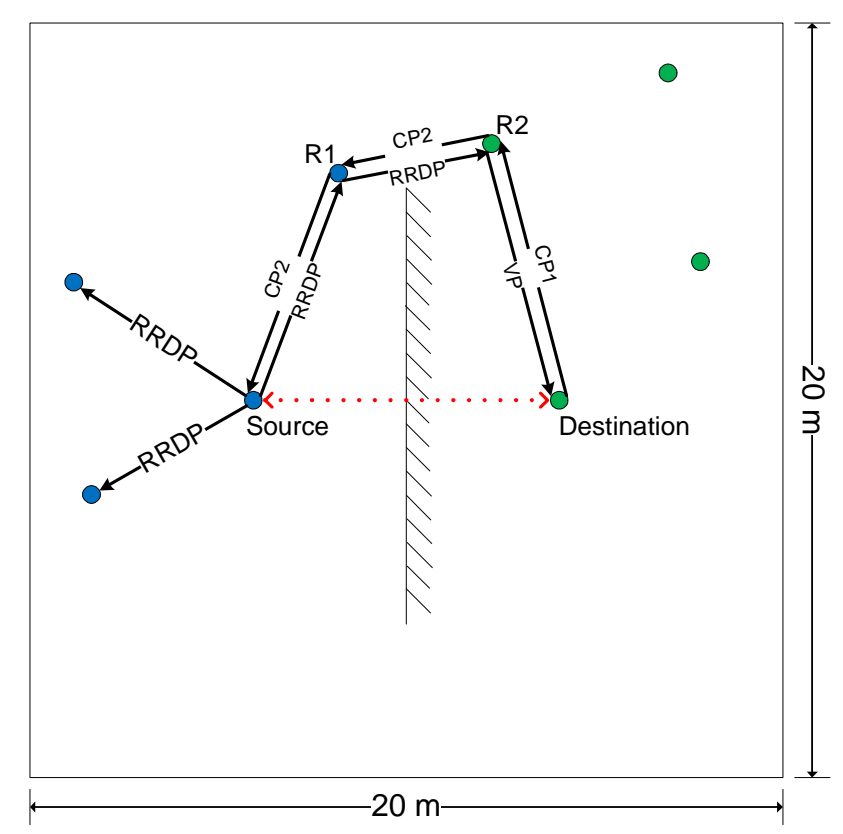

Figure 3: Peer-to-Peer protocol illustration

through new faces on both devices. If not, that means the interrupt is due to either out of range or blocking, both of them require additional steps. In the meantime, the destination node will also update its local neighbor table by sending out Neighbor Discovery Packet (NDP) with id information and depth count.

\subsection{2}

The source node first checks its own local table to see if a route already exists for the destination node. If yes, source sends validate packet to check and reestablish the link if link is valid.

\subsection{3}

If there is no such route in the local table or the path is no longer available, source sends Reactive Route Discovery Packet (RRDP) with preset forward depth count looking for rendezvous node which has the path to the destination node. If in a given period of time (associated with forward depth count) there is no response from any node, we consider that there is no such rendezvous node. Then the transmission terminates.

\subsection{4}

If a rendezvous node does exist, when it receives such RRDP, it will send out the same format of validate packet mentioned in step 2). And if there is no response, the source node entry will be deleted from rendezvous node's neighbor list. 


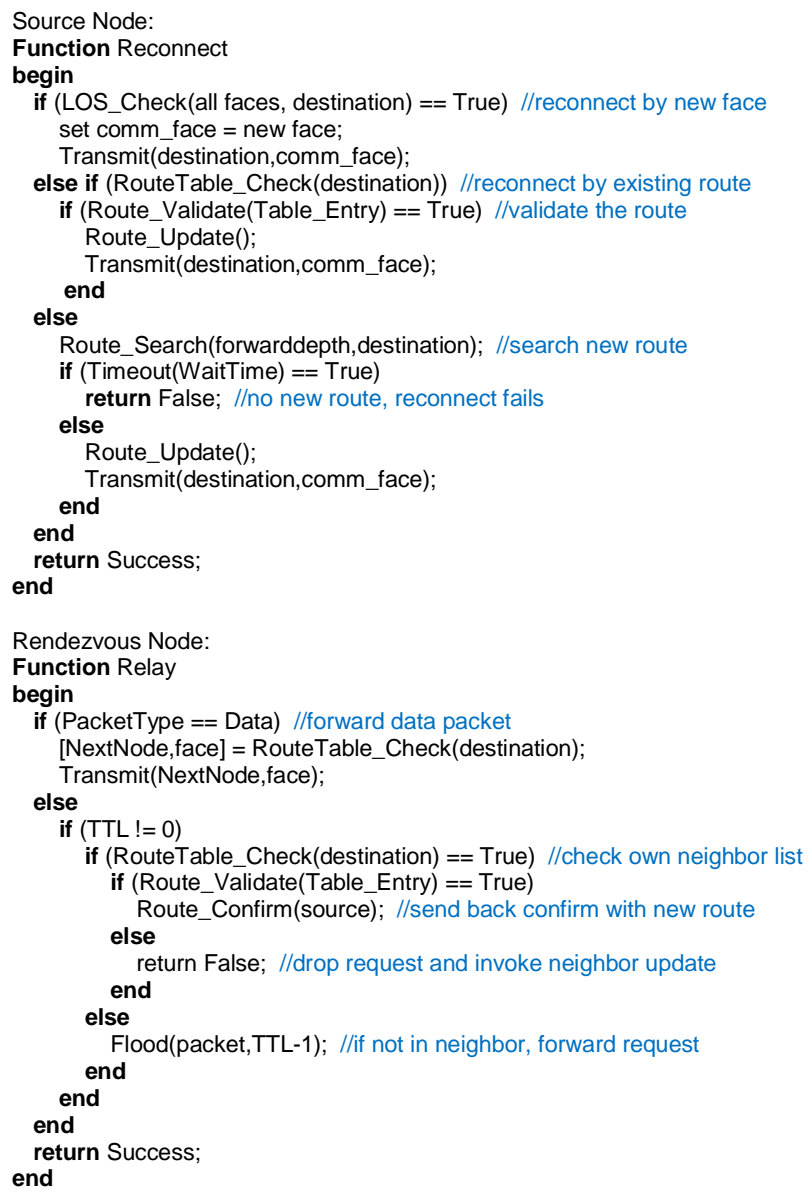

Figure 4: Pseudocode for Peer-to-Peer protocol

\subsection{5}

If all possible rendezvous nodes fail on validating the paths, the source will not be able to be notified in the given period of time and the transmission terminates. Otherwise, rendezvous nodes send back confirm packets with path information. The source node will examine and choose the best route to reconstruct the transmission.

The steps are illustrated in Fig. 3 and with pseudocode in Fig. 4.

\subsection{Peer-to-Host Protocol}

The other protocol includes hosts and the base stations at the ceiling level in our system for relaying the data. We consider the network as a two-layer geometry; nodes and base stations. Between every two peer nodes, there is only direct transmission and no multihop. Otherwise, the source node has to go through the host(s) to reach the destination node. We consider this in detail in the following steps. 


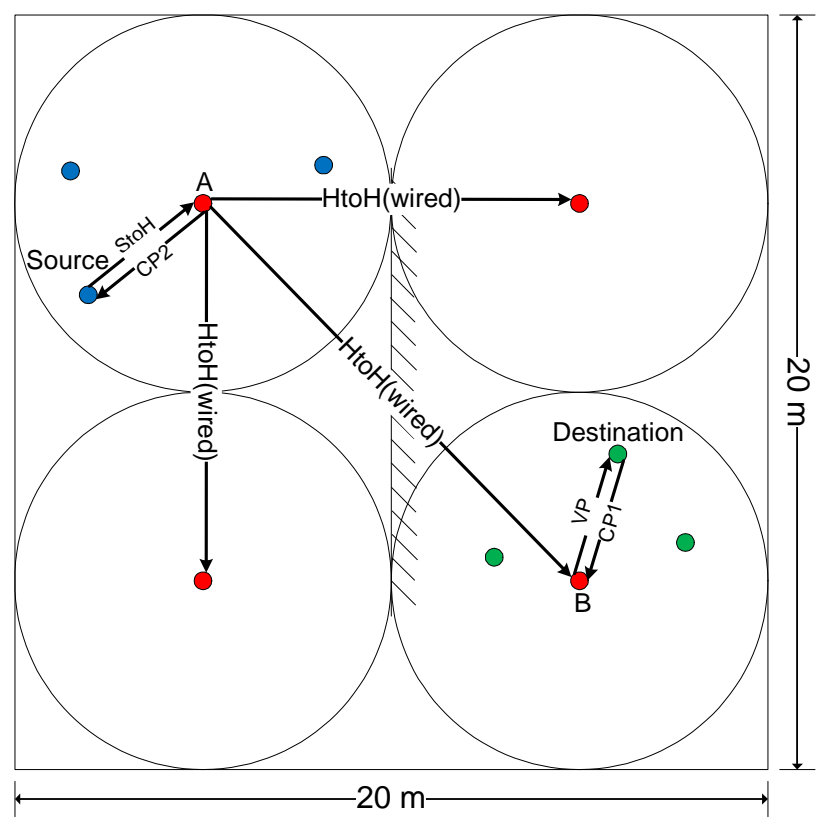

Figure 5: Peer-to-Host protocol illustration

\subsection{1}

The first step is very similar to that of the peer-to-peer protocol. The source node will first try to find alternative direct contact with destination node through other faces, and reestablish the link through new faces on both devices if available.

\subsection{2}

If there is no direct contact, source node will send a Source-to-Host (StoH) packet to its own host (Host A). The host then checks its node list to find out if the destination node is also under its coverage. If yes, a validate packet will be sent to check the availability.

\subsection{3}

If destination node is not in the list or there is no confirmation, host A will send out a similar request, Host-to-Host (HtoH) packet, to all its neighbor hosts in the local network (for example, all other ceiling lamps in the same office room).

\subsection{4}

Every peer host will check its own node list based on the information in HtoH. If the destination node exists, the corresponding host (Host B) will also need to check the link validation.

\subsection{5}

Similarly, if in a given period of time no response is sent back due to either no host has destination node in list or the link no longer exists, we consider the transmission terminated. Otherwise, the 


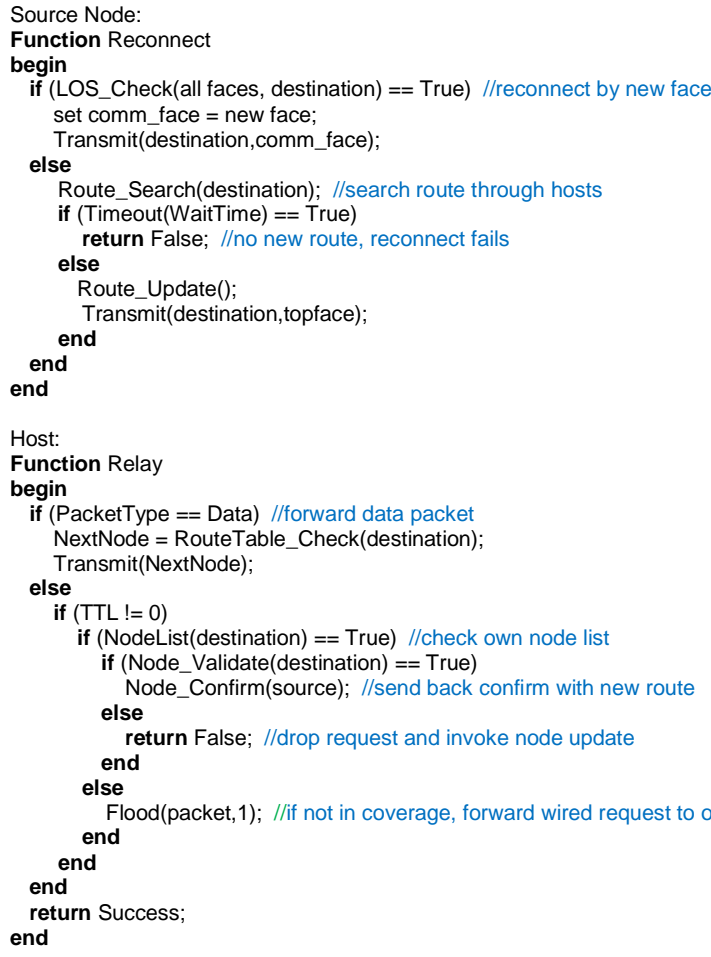

Figure 6: Pseudocode for Peer-to-Host protocol

destination node will confirm the link to B, and then B will confirm to A and source node, so that the link can be reestablished.

Similarly, the steps are illustrated in Fig. 5 and with pseudocode in Fig. 6.

\section{Connection and Rate Performance Analysis}

We first discuss connection performance by two simulations.

For the Peer-to-Peer protocol, the scenario we consider is a $20 \mathrm{~m}$ x $20 \mathrm{~m}$ room. The forward depth count is set to 2 , and the neighbor depth count is set to 1 . The communication range is a radius of $10 \mathrm{~m}$. We iterate 10,000 times. The transmission is between two nodes located at $(6,10)$ and $(14,10)$. The block is a wall from $(10,4)$ to $(10,16)$. We calculate a Reconnect Success Ratio for different numbers of users.

For the Peer-to-Host protocol, the simulation analysis is different. First, the nodes $(6,10)$ and $(14,10)$ are located close to the center of the room and they are not always under the coverage of the host. Therefore, the discussion of the reconnect success ratio between them becomes meaningless. Secondly, in the first simulation, if the two depth counts are chosen sufficiently large, the full connectivity can always be achieved. However, in Peer-to-Host protocol, only if the nodes are within the coverage of the host, will the full connectivity be achieved. Therefore, instead of Reconnect Success Ratio, we consider Fully Connectivity Ratio for this protocol. We use the 4 hosts in the same scenario and the coverage radius of each host of $5 \mathrm{~m}$, corresponding to half of the room side length. 
The two scenarios are illustrated in Fig. 3 and 5.

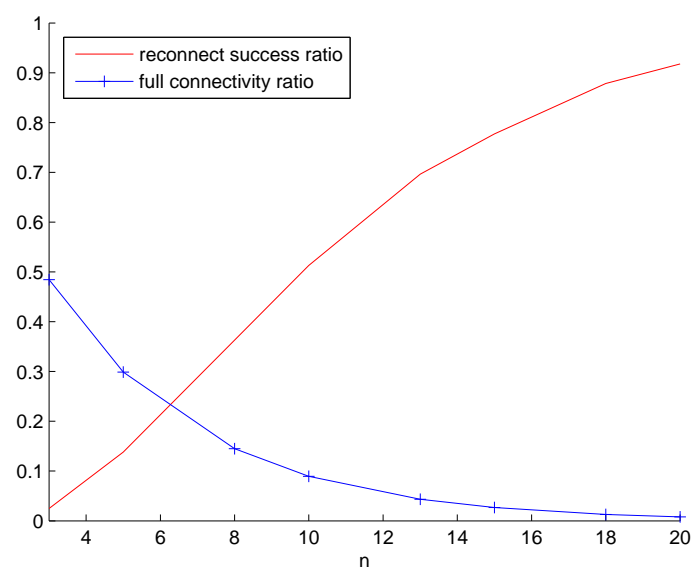

Figure 7: Reconnect ratio and full connectivity ratio for the two protocols

Fig. 7 shows that the peer-to-peer protocol needs more nodes to achieve high successful ratio. When the number of users reaches 20, the ratio is more than $90 \%$. However, it also increases additional packets and overheads that burden the system. For the network, the burden equals AverageEntry $* \sum_{k=0}^{\text {forwarddepth }-1}(\text { AverageEntry }-1)^{k}$. For example, in our simulation, when there are 20 users, the average entry is 7.67 neighbors, which makes the burden as high as 58.7 routing packets. Similarly, the burden to each node is the entry amount in the neighbor table. In our simulation, we only consider one depth neighbor which has an average of 7.67 neighbors for 20 users. If the depth becomes 2, this burden will increase to 13.1 neighbors.

In contrast, the figure shows that although peer-to-host protocol makes the architecture simpler (the burden to the whole network is always 1, and only the host needs to restore the entry information), the guarantee of full connectivity may not be good enough for a large number of users if hosts have limited coverage. In the peer-to-peer protocol, the node can increase the depth count to reach the destination, which however increases the complexity. But in peer-to-host protocol, the node in the shadow has no way to transmit information.

Since, we adopt CSMA/CA as multiple access solution, the discussion of real throughput performance will start with a theoretical model for CSMA/CA from [15]. By using this model and customizing it to our specific architecture, we can identify the packet transmission probability, $\tau$, and conditional collision probability, $p$. Considering a CSMA/CA with a contention window of $W$ and maximum backoff stage of $m$, from [15] we have

$$
\tau=\frac{2(1-2 p)}{(1-2 p)(W+1)+p W\left(1-(2 p)^{m}\right)} .
$$

We consider the worst case that every node always has a packet to deliver. For the uplink of node to host communication, if more than one node chooses the current time slot to transmit, collision will occur at the host. So, for $n$ nodes,

$$
p=1-(1-\tau)^{n-1} .
$$


For node-to-node ( $\mathrm{p} 2 \mathrm{p})$ communication, the analysis is more complex. We know that the user device has six faces, so the transmission from nodes which are not within the FOV of face sending the packet are not going to interfere. Even for the node within that FOV, if they don't have packet to transmit at the same time slot, the collision will not occur. Therefore, the new collision probability is

$$
\begin{aligned}
p & =1-\sum_{k=0}^{n-1}\left(\begin{array}{c}
n-1 \\
k
\end{array}\right)\left(\frac{5}{6}\right)^{n-1-k}\left(\frac{1}{6}(1-\tau)\right)^{k} \\
& =1-\left(1-\frac{1}{6} \tau\right)^{n-1} .
\end{aligned}
$$

By solving these two formulas we are able to have a unique pair of results for $\tau, p$. Before evaluating the throughput, we need to define the time variables. Based on 802.11 MAC specifications, we set them as in Table 1 .

Table 1: Time variables definition [15]

\begin{tabular}{|c|c|}
\hline Payload size & 8184 bits \\
\hline MAC header & 272 bits \\
\hline PHY header & 128 bits \\
\hline ACK & 112 bits + PHY header \\
\hline RTS & 160 bits + PHY header \\
\hline CTS & 112 bits + PHY header \\
\hline Propagation delay $(\delta)$ & $1 \mu \mathrm{s}$ \\
\hline Slot time $(\sigma)$ & $50 \mu \mathrm{s}$ \\
\hline SIFS & $28 \mu \mathrm{s}$ \\
\hline DIFS & $128 \mu \mathrm{s}$ \\
\hline
\end{tabular}

There are three cases for any time in the transmission procedure; empty time slot when every node is in the backoff contention window, failed transmission when there are more than one nodes sending out the RTS, and successful transmission when only one node is trying to send out the RTS. Therefore, based on CSMA/CA scheme, reference [15] shows

$$
\begin{aligned}
T_{\text {succ }}= & \frac{R T S}{\text { rate }}+\text { SIFS }+\delta+\frac{C T S}{\text { rate }}+S I F S+\delta+\frac{\text { Header }}{\text { rate }} \\
& +\frac{\text { Payload }}{\text { rate }}+S I F S+\delta+\frac{A C K}{\text { rate }}+D I F S+\delta, \\
T_{\text {fail }}= & \frac{\text { RTS }}{\text { rate }}+D I F S+\delta .
\end{aligned}
$$

We define normalized throughput as the ratio of real statistical rate, which is the average device throughput under worst case, over the capacity the device. Therefore, we have our formula for it:

$$
S=\frac{n \tau(1-\tau)^{n-1}(\text { Header }+ \text { Payload }) / \text { rate }}{(1-\tau)^{n} \sigma+n \tau(1-\tau)^{n-1} T_{\text {succ }}+\left(1-(1-\tau)^{n}-n \tau(1-\tau)^{n-1}\right) T_{\text {fail }}} .
$$


Considering the real throughput, for uplink transmission, we need to multiply $S$ with device capacity (maximum rate) and for total throughput of node-to-node links, further multiply the number of faces on each device, since all faces can work in parallell without interfering with each other.

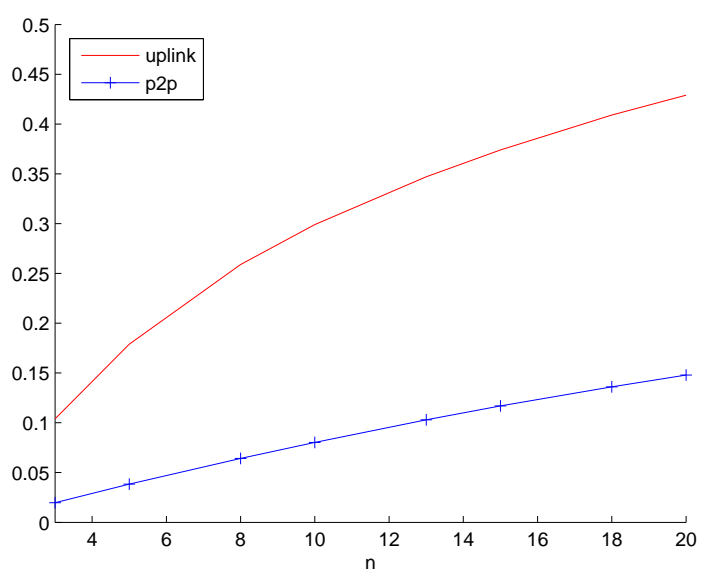

Figure 8: Collision rates

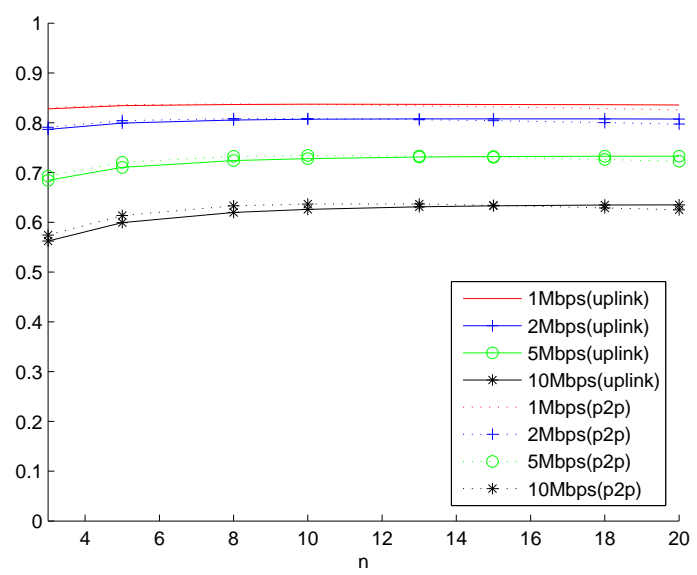

Figure 9: Normalized throughput of system

By splitting the horizon into 6 parts, the probability of collision can be greatly reduced as shown in Fig. 8. This is because the area can introducing collisions has been reduced to one sixth. In Fig. 9, the node-to-node scheme, however, doesn't give much efficiency boost over uplink transmission. This is because $\tau$ is not very large so that its increase does not substantially improve the overall system performance. Also, we see that high speed can result low efficiency since the time ratio of payload will be decreased by increasing the rate. Even though, due to the parallel transmission ability, the real throughput can still be greatly improved. We consider the rate capacities for uplink and node-to-node transmission to be 2 Mbps and $10 \mathrm{Mbps}$ respectively. In Fig. 10, the result shows that for uplink transmission in the four user case, each user can have an average rate of $422 \mathrm{kbps}$, and for node-to-node communication, the average rate is over $9 \mathrm{Mbps}$ giving the 


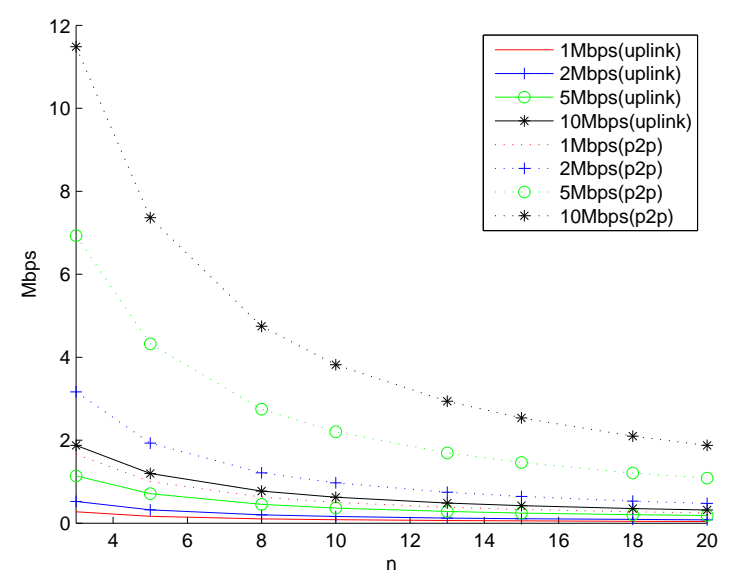

Figure 10: Real throughput of user

transmission on every face a minimum rate in excess of $1.5 \mathrm{Mbps}$. Remembering that the performance is calculated under the worst case scenario in which every node always has packets to send, therefore the results represent the lower bounds of the performance.

Based on all above, Table 2 generalizes our observations.

Table 2: Comparison of two protocols

\begin{tabular}{|c|cc|}
\hline Performance & Peer-to-peer & Peer-to-host \\
\hline Complexity & High & Low \\
\hline Overhead & High & Low \\
\hline Mobility & Low & Medium \\
\hline Speed & High & Low \\
\hline Interference & Low & High \\
\hline Burden to Host & No & Yes \\
\hline Outdoor Extension & Yes & No \\
\hline
\end{tabular}

\section{Conclusions}

In this paper we propose a next generation FSO system using visible light that exploits relaying and multiple access with a hexagonal line-of-site transceiver configuration. We introduce two network solutions for the LOS problem. From the discussion in previous section, we know that both protocols have advantages and disadvantages. The peer-to-peer protocol leverages a narrow beam and field of view from the proposed device and thereby can have good performance in terms of speed without a central host. The peer-to-host protocol, in contrast, is simpler and easy to implement, but due to the diffuse link model and interference, is less amenable to high data rates and requires a host to be available.

The adoption of each protocol depends on the desired behavior of the communication model. 
When the application requires transferring large data, the first protocol is most appropriate. If the application produces short bursts of data or the data rate requirements are relaxed as in many industrial automation scenarios, then the second protocol is a good choice. It is simpler and can readily support mobility of devices. Applications like in-office P2P messaging, in-building location services and the like can use the second protocol.

\section{References}

[1] T. Mukai and S. Nakamura, "White and UV LEDs," Oyo Buturi, vol. 68, no. 2, pp. 152-155, 1999.

[2] J. M. Kahn and J. R. Barry, "Wireless Infrared Communications," Proceedings of IEEE, 1997. 85(2): p. 265-298.

[3] J. B. Carruthers, "Wireless Infrared Communications," Wiley Encyclopedia of Telecommunications, 2002.

[4] S. Hrarilovic, "Wireless Optical Communication Systems," Springer Science, 2005.

[5] S. Arnon, "Optical Wireless Communication," Encyclopedia of Optical Engineering, 2003.

[6] A. C. Boucouvalas, "Challenges in Optical Communications," Optics \& Photonics News, 2005.

[7] G. Pang, T. Kwan, H. Liu and C. Chan, "LED Wireless: A Novel Use of LEDs to Transmit Audio and Digital Signal," IEEE Industry Applications Magazine, 2002.

[8] T. Douseki, "A Batteryless Optical-wireless System with White-LED Illumination," 15th IEEE International Symposium on Personal, Indoor and Mobile Radio Communications, 2004.

[9] M. Wada, T. Yendo, T. Fujii and M. Tanimoto, "Road-to-vehicle Communication Using LED Traffic Light,” Proceedings IEEE Intelligent Vehicles Symposium, June, 2005.

[10] S. Hranilovic and F. R. Kschischang, "A Pixelated MIMO Wireless Optical Communication System," IEEE Journal of Selected Topics in Quantum Electronics, VOL. 12, NO. 4, JULY/AUGUST 2006.

[11] Hoa Le Minh, D. O’Brien, G. Faulkner, L. Zeng, K. Lee, D. Jung, Y. Oh and Eun Tae Won, "100-Mb/s NRZ Visible Light Communications Using a Postequalized White LED," Proceedings IEEE Photonics Technology Letters, August, 2009.

[12] T.D.C. Little, P. Dib, K. Shah, N. Barraford and B. Gallagher, "Using LED Lighting for Ubiquitous Indoor Wireless Networking," IEEE International Conference on Wireless \& Mobile Computing, Networking \& Communication, Oct., 2008.

[13] Y. Alqudah and M. Kavehrad and S. Jivkova, "Optical Wireless Multi-Spot Diffusing; a MIMO Configuration," IEEE International Conference on Communications, Volume 6, 20-24 June 2004. 
[14] M. Yuksel, J. Akella, S. Kalyanaraman and P. Dutta, "Free-space-optical Mobile Ad Hoc Networks: Auto-configurable Building Blocks," ACM/Springer Wireless Networks, Volume 15, Number 3, pages 295-312, April 2009.

[15] G. Bianchi, "Performance Analysis of the IEEE 802.11 Distributed Coordination Function," IEEE Journal on Selected Areas in Communications, Volume 18, Mar. 2000. 